\title{
Theoretical limits on the efficiency of a quantum vacuum thruster
}

\author{
G. Modanese \\ Free University of Bolzano, Faculty of Science and Technology, P.za Università 5, Bolzano, Italy
}

\section{Email address:}

Giovanni.Modanese@unibz.it

\section{To cite this article:}

G. Modanese. Theoretical Limits on the Efficiency of a Quantum Vacuum Thruster. International Journal of Astrophysics and Space Science. Special Issue: Quantum Vacuum, Fundamental Arena of the Universe: Models, Applications and Perspectives.

Vol. 2, No. 6-1, 2014, pp. 39-45. doi: 10.11648/j.jjass.s.2014020601.15

\begin{abstract}
Theory and experiments show that vacuum fluctuations in quantum gravity can be abnormally strong, also at the micrometer or nanometer scale, for the following reasons: (1) the Einstein action is not positive-definite; (2) it is the only possible effective gravitational action; (3) quantum mechanics, in the form of the Feynman path integral, must apply to it, because any natural process is the result of all its possible quantum amplitudes; (4) due to (1), there are important non-classical virtual gravitational field configurations which can agree on a common phase. These field configurations can only interact directly with coherent matter, but can emit virtual gravitons which are absorbed by ordinary matter. All this makes possible, in principle, a vacuum thruster much more efficient than those based on the electromagnetic Casimir effect. We give an estimate of its efficiency based on the mentioned microscopic processes and on some parameters observed in experiments on anomalous forces with superconductors. With the observed energy efficiency of the order of $10^{-4}$ and an electric power of $10 \mathrm{~W}$ per kilogram of mass to propel, we find that a velocity of $0.1 c$ can be reached in ca. 10 years. Possible improvements and practical limits are outlined. We discuss how the concept of ideal vacuum should be modified in order to allow a consistent description of these phenomena.
\end{abstract}

Keywords: Quantum Gravity, Vacuum Fluctuations, Superconductors, Propellantless Thruster

\section{Introduction}

\subsection{The Quantum Vacuum}

In modern physics the vacuum state, i.e. the zero-energy and zero-momentum state from which all particles and fields have been removed, and which looks exactly the same to any moving observer, has non-trivial dynamical properties which affect several processes. The reasons for this are both formal and physical, and are explained in many textbooks and articles. A good introduction, with reference to the concept of zero-point energy of the elementary quantum field oscillators, can be found in $[1,2,3]$. These works then proceed to discuss the Casimir effect, considered to be a manifestation of the reality of the zero-point electromagnetic vacuum fluctuations, and explore the possibility of a "dynamical Casimir effect" which generates real photons from the vacuum (more about this below).

Another common approach to the properties of the quantum vacuum is the investigation of vacuum polarization effects and "radiative" corrections to quantum processes, like the Lamb shift in hydrogen (another tiny vacuum effect that has been confirmed experimentally [2]). It is a general property of quantum mechanics that the amplitude of a process is the sum of the amplitudes of all possible alternatives leading from the initial to the final state. Therefore for any scattering process or any Feynman diagram describing an elementary particle interaction one can imagine that there are intermediate steps involving the creation and re-annihilation of virtual particles with very short lifetime. For example, according to the time-energy uncertainty principle $\Delta E \Delta t \approx \hbar \approx 10^{-34} \mathrm{Js}$, a virtual electron-positron couple, with energy ca. equal to $1 \mathrm{MeV}$, has a lifetime of the order $10^{-21} \mathrm{~s}$. Formal perturbation theory allows to write down all these contributions in a systematic way. The virtual particle interpretation of these contributions is inspired by the visualization through Feynman diagrams and by analogies with polarization phenomena in real media.

In theories more complex than quantum electrodynamics, the properties of the vacuum resulting from this kind of analogies are quite exotic. For instance, in Yang-Mills theory the property of "asymptotic freedom" implies that the 
vacuum anti-shields charges (more exactly, the so-called U(1) charges), thus acting like a dielectric medium with dielectric constant $\varepsilon<1$ (in natural units). The quantum vacuum differs from an ordinary polarizable medium on a very important point: it is relativistically invariant. This implies that its relative magnetic permeability $\mu$ is related to $\varepsilon$ by $\mu \varepsilon=1 / \mathrm{c}^{2}$. It follows that the Yang-Mills vacuum has $\mu>1$ and is "paramagnetic" [4]. It should be stressed, however, that quantum field theory does not predict or allow any process in which a part of the energy-momentum of the initial particles is transferred to virtual particles and then to the vacuum, disappearing from the total energy-momentum balance. Actually, each time an experiment has shown an unbalance, theoreticians have successfully postulated the production of new undetected real particles, like in the case of neutrinos.

In this sense the vacuum appears to be a medium very different from, for instance, a crystal, where some well-known elementary scattering processes happen ("Umklapp processes") in which a part of the momentum is transferred to the medium. In the following we shall discuss if this property, well established for elementary particle scattering, may cease to be valid in processes involving coherent matter.

\subsection{Pioneers and Promoters}

The idea of "extracting from the vacuum" at least a small part of its huge zero-point energy has always been appealing. The cited paper by Maclay and Forward [2] considers to this end a modification of the Casimir effect in which electromagnetic radiation is emitted when an uncharged mirror is properly accelerated in the vacuum. The conclusion is, however, that the generated thrust is minuscule, so that this conceptual propulsion method is no more effective than a warp drive or a photon rocket. Some related proposals are briefly mentioned in [5] in connection with the experimental program at NASA Eagleworks laboratory (see end of this Section).

A longstanding theoretical and experimental program concerning electromagnetic zero-point energy and applications of the Casimir effect for nanotechnology is that of Puthoff and collaborators [6], whose theoretical investigations also concerned the possible origin of inertia from zero-point fluctuations and the explanation of gravitational forces as induced by vacuum fluctuations following the earlier approach by Sakharov. A solid-state Casimir device described as a transducer of vacuum energy which can operate in a repetitive cycle was proposed by Pinto $[7,8]$.

Physical commonsense based on the second principle of thermodynamics appears to prevent any useful extraction of energy from the vacuum. After all, we are not able to extract thermal energy from real matter, like for instance heat from the atmosphere or the oceans, unless some gradient of temperature, pressure etc. is available (usually only at large scale). It is true that vacuum energy is not thermal, but many proposals for its extraction by creation of a local gradient essentially require a sort of Maxwell demon, which is in contrast with quantum mechanics.

I might be wrong, however, and the "thermodynamics of vacuum" is admittedly a complex subject, still in evolution $[9,10]$. In the history of physics, thermodynamics came after the development of steam engines, and even then, the theory of elementary reversible systems like the Carnot engine was certainly not a sufficient guide in the discovery of internal combustion engines and refrigerators! The lesson (to be applied also to our proposals in Sect. 2) is that with new complex phenomena, we cannot expect that our previous "general theories" describe and comprise everything.

This is clearly also the opinion of several pioneers in this field, who have rushed to establish companies, obtain patents and raise venture capital for their experiments. Some of these people have a background in academic research, some do not. Many mainstream academicians regard their work as heretical and dangerous, but I personally believe that $21^{\text {st }}$ century fundamental physics is not in such a good shape that it can afford to just strike down any heresy. The correct approach, I think, is that advocated by Millis in his attempts to "distinguish pioneers from promoters" [11].

Richard Feynman once said "For a successful technology, reality must take precedence over public relations, for nature cannot be fooled". I think Galileo Galilei would agree.

The internal combustion engine example shows that often the particular process is discovered before the general principles. But, could one object, if decades pass without any practical progress, doesn't this mean that the empirical attempts are in a dead end? (It seems, for instance, that many fruitless attempts have been done to build small standalone batteries that recharge through electromagnetic fluctuations.) Or could this mean that a large-scale attempt is needed, like it happened for nuclear fission etc.?

My theoretical research work since 1994 and my cooperation with several experimentalists has led me to the following conclusions $[12,13]$ :

- It is possible to exchange considerable amounts of momentum with the quantum vacuum, using an external energy source able to excite coherent matter with sufficient density. The exchange is brought about by strong, still little-known, gravitational vacuum fluctuations.

- At least two experiments $[14,15,16]$ have demonstrated this effect, which is much stronger than those predicted by quantum electrodynamics as a consequence of Casimir effect and vacuum polarization. The intrinsic limits of propulsion applications of these effects are discussed in Sect. 2.

- A large, coordinated effort is necessary, both at the experimental and theoretical level, for a better understanding and for practical optimization of the existing devices. The scientific community has reacted skeptically to the claims of Podkletnov, citing a lack of data which made replication difficult. In the case of Poher, however, there are abundant data and several public demonstrations have been made. And the two effects are clearly related to each other. 
- These effects, being in essence a form of "vacuum propulsion" $[2,6]$, may still be regarded as heretical or at least anomalous in the context of traditional physics, because they appear to violate both the momentum conservation principle (if one denies the possibility of momentum transfer to the vacuum) and the Equivalence Principle (since vacuum propulsion allows a closed, isolated system to move with different acceleration from the other test particles). Both principles have been previously verified in several experiments to a high degree of accuracy and are embodied in the main assumptions of quantum field theory and general relativity, which are in turn well established experimentally. Therefore in order to accommodate anomalous results, a better understanding of the concept of vacuum is needed, and of the possible difference between "ideal and real vacuum", and of the role that coherent matter plays in quantum field theory and quantum gravity.

We close this section mentioning other two experiments on vacuum thrusters.

In his accurate theoretical and experimental work with asymmetrical capacitors and "Mach thrusters", Woodward $[17,18]$ explains momentum exchange with the vacuum through an application of the Mach principle. We prefer a "local interpretation", namely we suppose that the momentum apparently missing from some microscopic processes is transmitted to gravitational vacuum fluctuations. This causes a small local perturbation of the homogeneity and isotropy of the vacuum. A complete formal description of this situation is still missing, as discussed in Sect. 2.

In a recent paper [19] White et al. report the generation of a small thrust $(\approx 40-91 \mu \mathrm{N})$ by RF cavities excited with a power of $17-28 \mathrm{~W}$. The paper cites previous experimental work by Y. Juan et al. and by G. Fetta. Comments on these results have appeared in several media [20]. Some scientists have expressed heavy criticism. Others have objected to the theoretical concept of "vacuum plasma" which is behind the experiment, stressing the fact that electromagnetic vacuum fluctuations are not like a plasma capable of magneto-hydrodynamic effects. In our opinion the paper should be regarded as preliminary, since the measurement campaign was brief and experiments of this kind are much prone to error.

\section{The Thruster and Its Efficiency}

\subsection{Quantum Gravity and Vacuum Fluctuations}

In Sect. 1 we have discussed possible applications of the vacuum fluctuations of the electromagnetic fields. In fact, electromagnetic fields are those we know best and those which we are able to manipulate. The same cannot be said for the other fundamental forces. The weak and strong nuclear forces have a very short range and act only in the interactions of elementary particles. There exist no artificial sources of these forces. Gravitational fields cannot be manipulated either, because they require very massive sources - typically at the astronomical level - in order to attain a significant intensity. Therefore in all usual laboratory circumstances the gravitational field is only an external field which cannot be influenced.

The Einstein gravitational field equations exhibit important non-linear effects, and therefore, unlike the Maxwell equations, they can have vacuum solutions which are not simply plane waves, but are like solitons or vortices in a fluid. (Compare for this the "geons" solutions summarized and generalized in [22].) Since, however, the fields of gravitational waves are very weak, non-linear effects of this sort are even weaker, at least at the classical level.

The role of the non-linearities of gravity in quantum theory is a much more difficult issue. In general, since energy gravitates, a gravitational field can "feed itself". One would then expect non-linear vacuum effects to be much more important in quantum gravity than in quantum electrodynamics, where in order to produce to produce vacuum polarization one needs at least the creation of couples of the lightest existing charged particles, namely electron/positron couples, which requires considerable energy. However, general dimensional considerations show that gravitational vacuum fluctuations should become important only at the Planck scale $L_{\mathrm{P}}\left(\approx 10^{-35} \mathrm{~m}\right)$, where they even involve changes in the topology of spacetime.

In fact, the problem of accounting for vacuum fluctuations in quantum gravity in a consistent way, "renormalizing" the theory, has prevented for a long time the unification of general relativity and quantum mechanics into a quantum theory of gravity comparable to quantum electrodynamics. Eventually, a consistent theory has been obtained, Loop Quantum Gravity, which aims at describing the quantum structure of spacetime down to distances of the order of $L_{\mathrm{P}}$, and has made some predictions for very high energy phenomena. On the other hand, it has gradually become apparent that at larger scales it is possible to formulate an effective theory of quantum gravity where the renormalization problem, if not eliminated, is made harmless [22].

A still open problem is that of the cosmological constant: we need to explain why the energy of the vacuum fluctuations of all quantum fields, including the gravitational field itself, does not generate a gravitational field, affecting the curvature of spacetime. Several different solutions to this paradox have been proposed, but none has been yet generally accepted. Personally, I find most convincing the non-perturbative quantum models of Hamber, Ambjorn and collaborators $[23,24]$, where a smooth, almost flat spacetime is the result of a global dynamical process and the cancellation of vacuum contributions occurs spontaneously through an "emergence" mechanism.

The reasons why we think that the gravitational vacuum fluctuations can be quite important at the practical level and even responsible for the anomalous effects described in [14] and for the exchange of momentum with the vacuum at the microscopic level have been explained in [13]. Let me recall them here briefly. While we cannot be sure that the Einstein 
action is so fundamental that it has to be quantized down to the Planck scale, we know for sure that it is the only relativistic invariant effective field action which can describe the gravitational field, as shown by Feynman and Weinberg on very general grounds [25]. Therefore the Einstein action must be quantized, at least at the atomic scale, if we want to apply it at that scale.

The most general way to quantize a theory is through the path integral approach. This approach can be very complicated from the mathematical point of view, but physically it expresses the very fundamental principle of quantum mechanics that the amplitude of any process is the sum of the amplitudes of all its different possible realizations. Now, for a typical relativistically invariant action which is positive-definite, like that of QED, only paths which are very close to the classical path contribute significantly to the Feynman path integral, while all other paths interfere wildly and essentially cancel out. In other words, quantum configurations which are very far from the classical configurations cannot "agree on a common phase" in the sum over paths, and so interfere negatively between themselves and in the end do not give a significant contribution to the overall amplitude of physical processes.

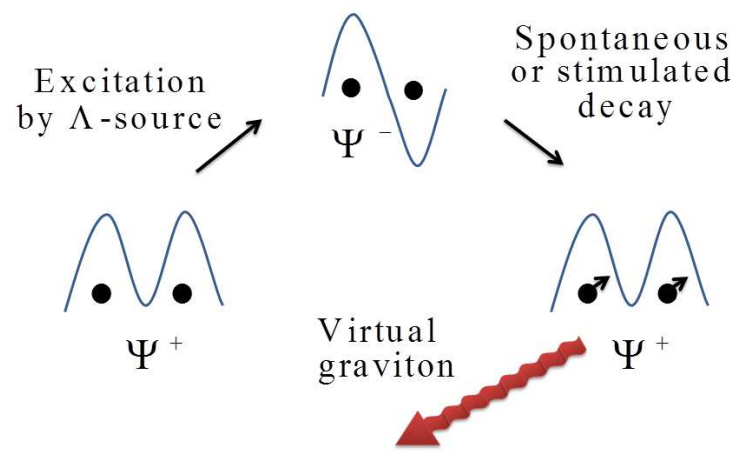

Figure 1. Microscopic model of anomalous radiation emission from superconductors. A couple of equal gravitational zero-modes can be excited by an oscillating coherent source with frequency $f$ and decays again to its symmetric ground state $\Psi^{+}$emitting a virtual graviton, which can propagate and transfer momentum $p$ to ordinary matter. The virtual masses recoil in the emission, and this amounts to a transfer of momentum $-p$ to the vacuum. The energy ( $\mathrm{hf}$ ) for the process comes from the coherent source. The ratio $(\mathrm{hf} / \mathrm{p})$ is of the order of $1 \mathrm{~m} / \mathrm{s}$.

In the path integral of quantum gravity, the situation is very different. Due to the non-positivity of the action, there exist vacuum quantum configurations which are very different from the classical configurations in vacuum. Besides gravitational waves, which make the action trivially zero because their scalar curvature vanishes at any point, there are static field configurations, called "zero-modes of the Einstein action", which have zero action thanks to a more complex mechanism of cancellation of the positive and negative contributions to scalar curvature in close but distinct regions of space. These field configurations have a small core, at whose center the metric component $g_{00}$ is zero, but outside the core the metric looks like a Schwarzschild metric, with negative mass. So they behave like virtual negative masses.
These vacuum fluctuations are determined in a crucial way by the non-linear terms in the field equations, they exist at all scales and their mass depends on the scale. On the scale relevant for our considerations (nanometers), they are of the order of $10^{-13} \mathrm{~kg}$, which is very large on the atomic mass scale. Like the vacuum fluctuations of QED, they are homogeneously and isotropically distributed, and therefore have no influence on processes involving elementary particles; but unlike the virtual electron/positron couples of QED, they last for a relatively long time and are not subject to the usual time-energy uncertainty relation.

Virtual masses interact gravitationally, forming weakly bound states, such that transitions between these states can be induced by a coherent source like a classical field or an order parameter of a quantum system, oscillating with frequency in the range of $\mathrm{MHz}$ to $\mathrm{THz}$ (Fig. 1). The excited bound states decay to the ground state emitting virtual gravitons, which are absorbed by surrounding "target" particles, with a net momentum transfer. The recoil momentum of the weakly bound virtual couple is eventually transferred to the vacuum. This mechanism is described in details in [13].

The macroscopic/integrated consequences of these microscopic interactions are quite complex, depending for instance on whether the momentum of the recoiling virtual particles is eventually transferred to matter, or not. We have discussed in [13] the several possible alternative macroscopic patterns, for comparison with the experimental results. The most plausible conclusion is that the alternative which is effectively realized is that called "emitter shooting asymmetrically on itself, recoil momentum dispersed in vacuum" (compare Fig. 2).

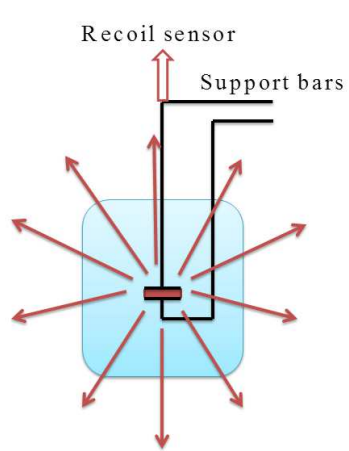

(a)

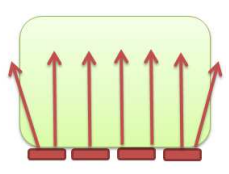

(b)
Figure 2. (a) Interpretation of the recoil in the laboratory experiments by Poher as due to an asymmetrical "shooting on itself" of the emitter (red). The emission of virtual gravitons (red arrows) is approximately isotropic, but the predominant effect on the support bars is in the upward direction. (b) If emitters are used for propulsion in space, then the virtual gravitons can only be emitted in a direction where they find payload matter to absorb them. This is likely to increase the overall efficiency.

Supposing this is the micro-to-macro process occurring in the experiments, let us evaluate its energy efficiency and its limitations. To this end we shall also exploit some crucial experimental data, namely the velocity of the targets, the frequency of the oscillating current and the electric energetic balance. So the strength of this estimate lies in its innovative 
microscopic interpretation, based on established fundamental principles of gravitation and quantum mechanics, and in the use of experimental data.

The weak points are, of course, that the experiments need confirmation and that the theory is in an initial stage. The existence of anomalous gravitational fluctuations has been proven mathematically, but we lack a conceptual framework in which we can show that the recoil of these fluctuations is a transfer of momentum to the vacuum state. This framework will probably require a distinction between an ideal vacuum state (such that the average $\langle 0|\mathrm{P}| 0\rangle$ of the total momentum is exactly equal to zero) and a "perturbed vacuum state". For all practical purposes the perturbed vacuum would not be distinguishable from the ideal vacuum state, because it acts as a momentum reservoir, in much the same way as a heat reservoir does not change its temperature in spite of absorbing or releasing some thermal energy.

Like the pioneers of thermal engines, we think we know the processes, but we still miss a rigorous general theory. In this situation, there is the lingering danger of imagining a process which goes against some general principle, like naive wannabe engineers planning a machine which executes a perpetual motion. Yet we are optimistic. The exchange of energy and momentum with the vacuum has been already proven in the context of the dynamical Casimir effect, although the exchanged amounts in that case are very small. Other possible theoretical approaches are mentioned in Sect. 3.

\subsection{Magnitude Order Estimates}

In [26] Millis has defined a "spacedrive" as a hypothetical propulsion device able to efficiently transform the potential energy of a source into kinetic energy, without the need of expelling any reaction mass. Fictional examples given by Millis to illustrate this concept include classical systems with components that are admittedly unphysical, like negative real masses or propulsive electric potential gradients generated locally by the spacecraft itself. He then proceeds to discuss possible benefits of such systems and future prospects for their realization.

Here we describe the working principle of a device with a low potential-to-kinetic energy conversion efficiency (of the order of $10^{-4}-10^{-3}$ ), based on a mechanism of repeated discharges as in [15], and which exchanges momentum with the vacuum fluctuations described in the previous section. We will only give order-of-magnitude estimates, since the real experimental situations are very complex, as discussed below.

Suppose that after each discharge an emitter, with mass of the order of $1 \mathrm{~kg}$, acquires a velocity of the order of $10^{-1} \mathrm{~m} / \mathrm{s}$, as observed by Poher, thanks to an asymmetric "shooting on itself" effect. Each new acceleration step can be re-computed in a new reference system at rest with the emitter. This is the crucial advantage of a vacuum process, which "dumps" momentum on vacuum fluctuations available, with the same features, in any moving system. Let us suppose that the final desired velocity is $0.1 c$; this is of course very large for standard propulsion methods, but still small enough to allow us, with good approximation, to sum all velocity variations disregarding relativistic effects.

With the mass and velocity step above, the kinetic energy required at the each discharge is of the order of $10^{-3} \mathrm{~J}$. For simplicity, consider a discharge duration of $10^{-5} \mathrm{~s}$ and suppose that the main frequency components of the current have frequency of the order of $10^{5} \mathrm{~Hz}$. This will excite virtual zero-mode states of energy $h f$ of the order of $10^{-29} \mathrm{~J}$, as explained in Sect. 1.2, quickly decaying into virtual gravitons of the same energy. The energy absorbed by the recoiling zero-modes in the emission is very small, because of their large virtual mass. Further admit that in the target each virtual graviton is absorbed by a nucleon, with mass of the order of $10^{-27} \mathrm{~kg}$, thus imparting it the observed velocity of $0.1 \mathrm{~m} / \mathrm{s}$. (This is a schematic lowest-order representation. In reality, the current pulse will have a broader frequency spectrum and the absorption of virtual gravitons in the target needs not to be in one-to-one correspondence with the nucleons, but there will be a continuum distribution.)

The energy efficiency of electric discharges in a superconductor is generally very low and we can assume an efficiency of $10^{-4}-10^{-3}$ for the excitation of zero-modes. This is one of the crucial difficulties encountered in the experiments. The impedance matching between the electric discharge circuit and the superconducting emitter is intrinsically poor and in an ideal superconductor the voltage drop $\Delta V$ during the discharge is very small. In real emitters it is probably of the order of $0.1 \mathrm{~V}$, compared to an applied voltage of thousands of volts; the exact figure is unknown, because any measurement is made very difficult by stray inductances. With a current $I \approx 10^{3} \mathrm{~A}$, this gives a power $P=I \Delta V \approx 10^{-2} \mathrm{~W}$ and a total kinetic energy $U=P \Delta t \approx 10^{-3} \mathrm{~J}$ per discharge, as observed. Real emitters must contain a sufficient number of inter-grain and intrinsic Josephson junction to allow for this $\Delta V$ (i.e, for a small partial penetration of the electric field) without destroying the order parameter of the superconductor.

Next suppose, to fix the ideas, to scale up this energy balance to a spacecraft mass of $10^{4} \mathrm{~kg}$. The energy required for a single velocity increment of $0.1 \mathrm{~m} / \mathrm{s}$ is of the order of $10^{5} \mathrm{~J}$, or $10 \mathrm{~J}$ per kilogram. The total energy needed in order to reach a velocity of $0.1 c=3 \cdot 10^{7} \mathrm{~m} / \mathrm{s}$ is between $10^{13}$ and $10^{14} \mathrm{~J}$, corresponding to the energy obtained in the fission of ca. $1 \mathrm{~kg}$ of $\mathrm{U}^{235}$. This is a relatively small fraction of the mass of the payload, comparable with the corresponding quantity for a photon rocket. The total acceleration time depends on the repetition rate of the discharges. With a rate of 1 repetition per second one finds $3 \cdot 10^{8} \mathrm{~s}$ (10 years). This is about $10^{7}$ times smaller than the acceleration time required for the same mass by a photon rocket with the same power.

The strongest limitation appears to originate from the mass of the hardware needed to power all the emitters, supposing one needs one emitter for propelling each kilogram of mass, and each emitter hosts discharges of $10^{3} \mathrm{~A}$. An efficient and compact, low-inductance design would be required, such that many emitters are powered in series. The electric discharges might also be replaced by other high-frequency generators, 
though this would be a major departure from the original experiments, requiring careful theoretical evaluation. Another major complication encountered in the experiments is the skin effect on the emitters [14].

\section{A list of Past and Future Issues}

This paper intends to be more an essay than a technical paper, as can also be seen from the absence of mathematical equations. It is based, however, on several technical papers. In addition to those already cited, we would like to mention $[27,28,29,30]$. This last section contains a quick list of possible future developments.

1. In [29] we have discussed the conditions for the occurrence of stimulated emission, leading to impressive laser action as reported in [15]. These conditions are essentially (a) higher frequency of excitation, due to shorter pulses and possible plasma oscillations in the gas discharge; (b) melt-textured structure of the emitter. A coherent beam could be very useful, even at low power, for scanning materials or biological tissues [13].

2. It has been suggested that the YBCO superconducting emitter might be replaced with an emitter made of some superconducting metamaterial, characterized by macroscopic coherence also at room temperature. This is an interesting possibility, but the performance is largely unknown, and depends (like for superconductors) on the achievable density of the order parameter and on the impedance matching between the emitter and the discharge circuit.

3. What are the electric and magnetic field strengths required for a pumping effect comparable to that of superconductors? The local $\Lambda$-term, or vacuum energy term, which is able to excite the zero-modes, receives from superconductors with large density gradients contributions of the order of $10^{6}-10^{8} \mathrm{~J} / \mathrm{m}^{3}$. The electric and magnetic strengths required to achieve the same density are very large: $10^{9} \mathrm{~V} / \mathrm{m}$ and $10 \mathrm{~T}$, respectively. Note that the $\Lambda$-term must also oscillate with a frequency of at least $10^{6} \mathrm{~Hz}$, in order to efficiently excite the zero-modes. This appears to exclude any role of the pure $B$ field. An induction $E$ field may instead play a significant role [13].

4. Point (3) is not (yet) a suggestion for an anomalous coupling between gravitation and electromagnetism. Actually I would not like to enter this subject, which is desperately entangled due to the accumulation, over the years, of a large number of mythical experimental claims, crude theories which disregard previous art, etc. One example of these myths could be the "Schlicher thrusting antenna" ([31] and ref.s). The authors of [31] attempt a replication (pointing out the lack of data in the original papers and patent) and explain why this phenomenon is in contrast with the basic laws of classical electromagnetism. Other longstanding claims are harder to dismiss, like the Biefeld-Brown effect, which has certainly a component due to air ionization but has been replicated also in in insulating oil [32].

Even if we stick with official science and peer reviewed papers published by scientists with a proven record, there are plenty of mysteries concerning gravitational forces at all scales, and their relations to other fundamental forces and to dark matter and energy [33,34,35]. Although General Relativity has passed numerous experimental tests, it is quite inevitable that sooner or later it will need some extension. The point is, which of these extensions are relevant for practical applications? Following a different route, in this paper we have argued that experiments with superconductors like those of Podkletnov and Poher, which display effects much stronger than electrogravitic coupling, can be explained through standard General Relativity and quantum mechanics. We think that these effects have a tremendous potential for practical applications already in the short term, both in the high-power field of propulsion and in the low-power field of signal transmission and materials inspection.

\section{References}

[1] Maclay, G. Jordan, and Robert L. Forward. "A gedanken spacecraft that operates using the quantum vacuum (dynamic Casimir effect)." Foundations of Physics 34.3 (2004): 477-500.

[2] Maclay, G. Jordan. "Thrusting against the quantum vacuum." Frontiers of Propulsion Science, In: Lu FK, editor, Progress in Astronautics and Aeronautics, Reston, Va.: AIAA 227 (2008): 391-422.

[3] Maclay, G. Jordan. "Gedanken experiments with Casimir forces and vacuum energy." Physical Review A 82.3 (2010): 032106.

[4] Nielsen, N. K. "Asymptotic freedom as a spin effect." Am. J. Phys. 49.12 (1981): 1171.

[5] White, H., et al. "Eagleworks Laboratories: Advanced Propulsion Physics Research." (2011). NASA Technical Reports Server, Report JSC-CN-25207.

[6] Puthoff, H. E., and S. R. Little. "Engineering the zero-point field and polarizable vacuum for interstellar flight.", preprint arXiv:1012.5264 (2010).

[7] Pinto, F. "Engine cycle of an optically controlled vacuum energy transducer." Physical Review B 60.21 (1999): 14740.

[8] Pinto, F. "Method and apparatus for energy extraction." U.S. Patent No. 6,477,028. 5 Nov. 2002.

[9] Klinkhamer, F. R., and G. E. Volovik. "Self-tuning vacuum variable and cosmological constant." Physical Review D 77.8 (2008): 085015.

[10] Scandurra, M. (2001). Thermodynamic properties of the quantum vacuum. arXiv preprint hep-th/0104127.

[11] Millis, Marc G. "Progress in revolutionary propulsion physics", preprint arXiv:1101.1063 (2011).

[12] Modanese, G., and G. A. Robertson, eds. Gravity-superconductors interactions: theory and experiment. Bentham Science Publishers, 2012.

[13] Modanese, G. "Gravity-Superconductors Interactions as a Possible Means to Exchange Momentum with the Vacuum." J. Space Exploration 3.2 (2014), arXiv:1408.1636. 
[14] Modanese, G. "A Comparison Between the YBCO Discharge Experiments by E. Podkletnov and C. Poher, and their Theoretical Interpretations", Appl. Phys. Res. 6 (2013) 59-73.

[15] E. Podkletnov and G. Modanese, Investigation of high voltage discharges in low pressure gases through large ceramic superconducting electrodes. J. Low Temp. Phys. 2003; 132: 239-259.

[16] C. Poher and D. Poher, Physical Phenomena Observed during Strong Electric Discharges into Layered Y123 Superconducting Devices at 77 K, Appl. Phys. Res. 3 (2011) 51-66

[17] Woodward, James F. (October 2004). "Flux Capacitors and the Origin of Inertia". Foundations of Physics 34 (10): 1475-1514.

[18] Fearn, H., \& Woodward, J. F. (2012). Recent Results of an Investigation of Mach Effect Thrusters. 48th AIAA/ASME/SAE/ASEE Joint Propulsion Conference \& Exhibit 30 July - 01 August 2012, Atlanta, Georgia AIAA 2012-3861

[19] D.A. Brady, H.G. White, P. March, J.T. Lawrence, F.J. Davies, "Anomalous Thrust Production from an RF Test Device Measured on a Low-Thrust Torsion Pendulum". http://ntrs.nasa.gov/search.jsp?R=20140006052

[20] September 2014 Newsletter, TauZero Foundation, www.tauzero.aero. J. Baez, Google+ posts, Aug. 2 and 3, 2014 https://plus.google.com/117663015413546257905/posts/C7vx $2 \mathrm{G} 85 \mathrm{kr} 4$

[21] Hartnett, G. S., \& Horowitz, G. T. (2013). Geons and spin-2 condensates in the AdS soliton. Journal of High Energy Physics, 2013(1), 1-12.

[22] Burgess, C. P. (2004). Quantum gravity in everyday life: General relativity as an effective field theory. Living Rev. Rel, 7(5), 3 .

[23] Hamber HW. Quantum Gravitation. The Feynman Path Integral Approach. Berlin: Springer 2009.
[24] J. Ambjorn, J. Jurkiewicz and R. Loll, Emergence of a 4D world from causal quantum gravity, Phys. Rev. Lett. 93 (2004) 131301.

[25] Alvarez, E. Quantum gravity: an introduction to some recent results. Reviews of Modern Physics 61.3 (1989) 561.

[26] Millis, M. 1997. Challenge to create the space drive. AIAA J. Propulsion Power 13(5): 577-582.

[27] Modanese G. The vacuum state of quantum gravity contains large virtual masses. Class. Quantum Grav. 2007; 24:1899-1909.

[28] Modanese G., Junker T. Conditions for stimulated emission in anomalous gravity-superconductors interactions. In: Classical and Quantum Gravity Research, Ed.s Christiansen MN, Rasmussen TK. Nova Science Publishers 2008; pp. 245-269.

[29] G. Modanese; Quantum Gravity Evaluation of Stimulated Graviton Emission in Superconductors. In: Modanese G., Robertson G.A., Ed.s., Gravity-Superconductors Interactions: Theory and Experiment, Ch. 5, Bentham (2012)

[30] G. Modanese; Anomalous gravitational vacuum fluctuations which act as virtual oscillating dipoles. In: Quantum Gravity, R. Sobreiro Ed., Ch. 1, InTech (2012)

[31] Fralick, G.C., and J.M. Niedra. Experimental results of Schlicher's thrusting antenna. Report NASA/TM-2001 -211207, November 2001, AIAA-2001-3657.

[32] Musha, T. Explanation of dynamical Biefeld-Brown Effect from the standpoint of ZPF field. JBIS, 61 (2008) 379-384.

[33] W. Dröscher. Reality of Gravity-Like Fields? Part I: Recent Experiments that Challenge Current Physics. J. Space Expl. 3 (2014)

[34] Hauser J. Reality of Gravity-Like Fields? Part II: Analysis of gravitomagnetic experiments. J. Space Expl. 3 (2014)

[35] Hauser J. The Physics of "Interstellar" - Mission Impossible. To appear in J. Space Expl., 2015. 